\title{
HARMONIC MORPHISMS FROM EVEN-DIMENSIONAL HYPERBOLIC SPACES
}

\author{
MARTIN SVENSSON
}

\begin{abstract}
In this paper we give a method for constructing complex valued harmonic morphisms in some pseudo-Riemannian manifolds using a parametrization of isotropic subbundles of the complexified tangent bundle. As a result we construct the first known examples of complex valued harmonic morphisms in real hyperbolic spaces of even dimension not equal to 4 which do not have totally geodesic fibres.
\end{abstract}

\section{Introduction}

A harmonic morphism between two pseudo-Riemannian manifolds is a map which is both harmonic and horizontally conformal, see [6] and [7]. Together these conditions form an over-determined non-linear system of partial differential equations. Various geometrical methods have been applied to construct examples of harmonic morphisms in different contexts.

Regarding locally defined harmonic morphisms in the real 3 and 4-dimensional hyperbolic spaces with values in a surface, a full classification has been known for some time, see [3] and [1]. This is also the case for locally defined (submersive) harmonic morphisms with totally geodesic fibres from any real hyperbolic space into a surface, see [9] and [2, Chapter 6]. Examples of locally defined harmonic morphisms without totally geodesic fibres from odd-dimensional real hyperbolic spaces into the complex plane have been constructed in [9] and [10]. Thus remains the case of locally defined harmonic morphisms without totally geodesic fibres from even-dimensional real hyperbolic spaces into surfaces. In this paper we give a method for constructing examples of such maps.

As an example we have the following: Let $\mathrm{R} H^{6}$ be embedded in the 7dimensional Minkowski space in the standard way. Then the restriction to $\mathrm{R} H^{6}$ of the complex valued map

$$
x \mapsto \frac{\left(x_{3}+x_{5}+i\left(x_{6}-x_{4}\right)\right)\left(2 x_{7}-x_{5}+i\left(2 x_{4}+x_{6}\right)\right)}{\left(x_{1}-x_{2}\right)^{2}},
$$

Received November 1, 2001. 
is a harmonic morphism. For the sake of generality, the setting will here be a bit more general and we will show how the method applies to any pseudoRiemannian space form of constant negative curvature.

When the codomain for a map from a pseudo-Riemannian manifold is the complex plane, the horizontal conformality is equivalent to the fact that the complex gradient is isotropic, that is, the square of the gradient vanishes. To construct examples of complex valued harmonic morphisms one could therefore look for functions whose complex gradients lie in some isotropic subbundle of the complexified tangent bundle.

On a Riemannian manifold, there is a one-to-one correspondence between maximal isotropic subbundles of the complexified tangent bundle and almost Hermitian structures, see e.g. [4]. Given an almost Hermitian structure $J$ on the Riemannian manifold $(M, g)$, we get a maximal isotropic subbundle of $T M \otimes C$ from its $(1,0)$-bundle. On the other hand, given such a maximal isotropic subbundle, we can clearly reconstruct $J$ by requiring it to act on this bundle by multiplication of $i$.

This connection between maximal isotropic subbundles and almost Hermitian structures is used by Baird and Wood in [4] and [5] to construct examples of complex valued horizontally conformal maps in Euclidean spaces, namely maps that are holomorphic with respect to some almost Hermitian structure. Further conditions imply harmonicity of the maps and thus give harmonic morphisms. Moreover, a parametrization of the almost Hermitian structures on open subsets of $\mathbf{R}^{2 m}$ gives conditions on all locally defined complex valued harmonic morphisms in $\mathbf{R}^{2 m}$ which are holomorphic with respect to some Hermitian structure ([4, Proposition 3.18]).

In the pseudo-Riemannian case, the situation is rather different since there may be isotropic subbundles even in the tangent bundle. The corresponding complexifications can therefore not arise from any almost Hermitian structure. In this paper we thus investigate similar constructions to those of Baird and Wood in pseudo-Euclidean space, using the notion of isotropic subbundles instead of almost Hermitian structures. Using a method of descent, we have means to construct complex valued harmonic morphisms from open subsets of pseudo-Riemannian space forms of constant negative curvature.

\section{Harmonic morphisms in pseudo-Riemannian geometry}

The main reference for harmonic morphisms between pseudo-Riemannian manifolds is the article [7] by Fuglede. Recall that a pseudo-Riemannian manifold is a smooth manifold $M$ endowed with a non-degenerate type $(0,2)$ tensor field $g$ of constant index, see e.g. [12]. We refer to $g$ as the metric tensor. For 
a subspace $\mathscr{K}$ of $T_{x} M$, we define

$$
\mathscr{K}^{\perp}=\left\{v \in T_{x} M \mid g(v, w)=0 \text { for all } w \in \mathscr{K}\right\} .
$$

If $\varphi: M \rightarrow N$ is a map, we denote by $\mathscr{V}_{x}$ the subspace $\operatorname{ker} d \varphi_{x}$ of $T_{x} M$ and $\mathscr{H}_{x}=\mathscr{V}_{x}^{\perp}$

DEFINITION 2.1. If $(M, g)$ and $(N, h)$ are pseudo-Riemannian manifolds and $\varphi: M \rightarrow N$ a smooth map, then $\varphi$ is said to be horizontally conformal if for any $x \in M$, either $\mathscr{H}_{x} \subseteq \mathscr{V}_{x}$ or the restriction of $d \varphi_{x}$ to $\mathscr{H}_{x}$ is conformal and surjective onto $T_{\varphi(x)} N$.

Recall that a map $\varphi:(M, g) \rightarrow(N, h)$ between two pseudo-Riemannian manifolds is said to be harmonic if

$$
\operatorname{trace} \nabla d \varphi=0 \text {, }
$$

where $d \varphi$ is considered as a section of the bundle $T^{*} M \otimes \varphi^{-1} T N$ with its natural connection, and the trace is taken with respect to $g$. In particular, a complex valued function on $M$ is a harmonic map if and only if it is a harmonic function, using the Laplace-Beltrami operator on $(M, g)$.

Definition 2.2. A map $\varphi:(M, g) \rightarrow(N, h)$ is said to be a harmonic morphism if for any locally defined harmonic function $f$ on $N$, the composition $f \circ \varphi$ is harmonic where defined on $M$.

Using a result of Hörmander, Fuglede generalized the following fact to pseudo-Riemannian geometry.

Theorem 2.3 ([7]). A smooth map $\varphi:(M, g) \rightarrow(N, h)$ between pseudoRiemannian manifolds is a harmonic morphism if and only if it is a horizontally conformal harmonic map.

The following result will be used throughout this paper.

Proposition 2.4. If $(M, g)$ is a pseudo-Riemannian manifold and $f$ : $M \rightarrow \mathrm{C}$ a function, then $f$ is horizontally conformal if and only if

$$
g(\nabla f, \nabla f)=0 .
$$

Here $g$ denotes the complex bi-linear extension of the metric tensor acting on complex vectors.

Proof. If we write $f=f_{1}+i f_{2}$ in the standard coordinates for $\mathrm{C}$ and fix $x \in M$, then

$$
\mathscr{H}_{x}=\operatorname{span}_{\mathrm{R}}\left\{\nabla f_{1}, \nabla f_{2}\right\},
$$


where the gradients are taken at the point $x$. Note that equation (1) is equivalent to

$$
g\left(\nabla f_{1}, \nabla f_{1}\right)=g\left(\nabla f_{2}, \nabla f_{2}\right), \quad g\left(\nabla f_{1}, \nabla f_{2}\right)=0 .
$$

Assume at first that these equations hold. If $v \in \mathscr{H}_{x}, v=v_{1} \nabla f_{1}+v_{2} \nabla f_{2}$, then

$$
\begin{aligned}
\left|d f_{x}(v)\right|^{2} & =v_{1}^{2} g\left(\nabla f_{1}, \nabla f_{1}\right)^{2}+v_{2}^{2} g\left(\nabla f_{2}, \nabla f_{2}\right)^{2} \\
& =g(v, v) g\left(\nabla f_{1}, \nabla f_{1}\right) .
\end{aligned}
$$

If $\mathscr{H}_{x} \neq\{0\}$, assume that $\mathscr{H}_{x} \cap \mathscr{V}_{x}=\{0\}$. Then $d f$ is conformal and injective on $\mathscr{H}_{x}$ so $\nabla f_{1}$ and $\nabla f_{2}$ cannot be parallel. Hence $\operatorname{dim} \mathscr{H}_{x}=2$ and $d f$ is also surjective on $\mathscr{H}_{x}$. If on the other hand $\mathscr{H}_{x} \cap \mathscr{V}_{x} \neq\{0\}$, let $v \in \mathscr{H}_{x} \cap \mathscr{V}_{x}$ be non-zero, $v=v_{1} \nabla f_{1}+v_{2} \nabla f_{2}$ with $v_{1} \neq 0$, say. Then

$$
0=g\left(v, \nabla f_{1}\right)=v_{1} g\left(\nabla f_{1}, \nabla f_{1}\right),
$$

so that $\mathscr{H}_{x} \subseteq \mathscr{V}_{x}$ by equation (2). This shows that $f$ is horizontally conformal.

Conversely, assume that $f$ is horizontally conformal. Then there is a continuous function $\eta$ on $M$ such that

$$
d f(v) \cdot \overline{d f(w)}=\eta(x) g(v, w), \quad v, w \in \mathscr{H}_{x} .
$$

To simplify the notation, introduce functions

$$
a=g\left(\nabla f_{1}, \nabla f_{1}\right), \quad b=g\left(\nabla f_{2}, \nabla f_{2}\right), \quad c=g\left(\nabla f_{1}, \nabla f_{2}\right) .
$$

Applying $d f$ on the horizontal vectors $\nabla f_{1}$ and $\nabla f_{2}$ and using equation (3) gives

$$
\begin{aligned}
\eta a & =a^{2}+c^{2} \\
\eta b & =b^{2}+c^{2} \\
\eta c & =(a+b) c .
\end{aligned}
$$

If $\mathscr{H}_{x} \subseteq \mathscr{V}_{x}$ then trivially $a=b=c=0$. If on the other hand $d f$ is surjective and conformal on $\mathscr{H}_{x}$, assume for the sake of a contradiction that $c \neq 0$. Then from equations (4) $\eta=a+b$ and $a b=c^{2}$. This last equation means that

$$
g\left(c \nabla f_{1}-a \nabla f_{2}, \nabla f_{2}\right)=0 .
$$

Since trivially $g\left(c \nabla f_{1}-a \nabla f_{2}, \nabla f_{1}\right)=0$, it follows from the non-degeneracy of $g$ that $c \nabla f_{1}=a \nabla f_{2}$. Hence $\operatorname{dim} H_{x} \leq 1$ which is impossible if $d f$ is to be surjective on $\mathscr{H}_{x}$. We thus conclude that $c=0$ and since $a, b \neq 0$ we see from equations (4) that $a=b=\eta$. Thus equation (1) is satisfied. 


\section{Parametrization of isotropic subbundles in even-dimensional pseudo-Euclidean space}

In this section we show how to parameterize some isotropic subbundles and their connection to horizontally conformal maps.

Definition 3.1. If $(M, g)$ is a pseudo-Riemannian manifold and $\mathscr{F}$ is a subbundle of the complexified tangent bundle $T^{\mathrm{C}} M$, then $\mathscr{F}$ is called isotropic if

$$
g(u, v)=0,
$$

for all $u, v \in \mathscr{F}$. Here $g(u, v)$ is the complex extension of the metric acting on $u$ and $v$.

Let $\mathrm{R}_{d}^{n}=\left(\mathrm{R}^{n}, g_{d}\right)$ denote the $n$-dimensional pseudo-Euclidean space [12] with its standard metric of index $d$ :

$$
g_{d}(u, v)=-\sum_{l=1}^{d} u_{l} v_{l}+\sum_{l=d+1}^{n} u_{l} v_{l} .
$$

Let

$$
\mathcal{U}_{n}=\left\{x \in \mathrm{R}_{d}^{n} \mid g_{d}(x, x)<0\right\} .
$$

If we restrict the multiplicative action of $\mathrm{R}^{*}$ on $\mathrm{R}_{d}^{n}$ to $\mathscr{U}_{n}$, we get as the quotient the pseudo-Riemannian hyperbolic space $\mathrm{R} H_{d}^{n}$, see e.g. [12, page 67] where a different notation is used for these spaces.

From now on, we will use the following ranges for the indices:

$$
1 \leq b, l, r \leq d, \quad d+1 \leq i, j, k \leq n, \quad 1 \leq s, t \leq n, \quad 1 \leq \mu, v \leq m .
$$

The last set of indices will enter in the next section. To get suitable coordinates in $\mathrm{R}_{d}^{2 n}$, we write

$$
u_{l}=x_{2 l-1}-x_{2 l}, \quad v_{l}=x_{2 l-1}+x_{2 l}, \quad q_{s}=x_{2 s-1}+i x_{2 s} .
$$

Suppose we have an open set $U \subseteq \mathrm{R}_{d}^{2 n}$ and a map

$$
A: U \rightarrow \mathfrak{g l}(n)^{\mathrm{C}} .
$$

For any $x \in U$, let $\mathscr{F}_{x}$ denote the subspace of the complexified tangent bundle $T^{\mathrm{C}} \mathrm{R}_{d}^{2 n}$ defined by the equations (taken at the point $x$ )

$$
\begin{aligned}
& \xi_{l} \stackrel{\text { def }}{=} d u_{l}+\sum_{r=1}^{d} a_{l r} d v_{r}+\sum_{k=d+1}^{n} a_{l k} d \bar{q}_{k}=0, \\
& \xi_{j} \stackrel{\text { def }}{=} d q_{j}+\sum_{r=1}^{d} a_{j r} d v_{r}+\sum_{k=d+1}^{n} a_{j k} d \bar{q}_{k}=0 .
\end{aligned}
$$


An easy calculation shows that these forms are linearly independent at each point over the complex numbers, so $\mathscr{F}$ is a (complex) subbundle of $T^{\mathrm{C}} \mathrm{R}_{d}^{2 n}$ of dimension $n$.

The metric $g_{d}$ provides a complex linear isomorphism $\eta \mapsto \eta^{\sharp}$ from $T^{* \mathrm{C}} \mathrm{R}_{d}^{2 n}$ onto $T^{\mathrm{C}} \mathrm{R}_{d}^{2 n}$ :

$$
g_{d}\left(\eta^{\sharp}, Z\right)=\eta(Z), \quad Z \in T^{\mathrm{C}} \mathrm{R}_{d}^{2 n} .
$$

It is easy to see that we have the following:

$$
d u_{l}^{\sharp}=-2 \partial_{v_{l}}, \quad d v_{l}^{\sharp}=-2 \partial_{u_{l}}, \quad d q_{j}^{\sharp}=2 \partial_{\bar{q}_{j}}, \quad d \bar{q}_{j}^{\sharp}=2 \partial_{q_{j}} .
$$

If we define vector fields $X_{s}=\xi_{s}^{\sharp} / 2$, then

$$
\begin{aligned}
& g_{d}\left(X_{l}, X_{r}\right)=-\frac{1}{2}\left(a_{l r}+a_{r l}\right), \\
& g_{d}\left(X_{l}, X_{j}\right)=\frac{1}{2}\left(-a_{j l}+a_{l j}\right), \\
& g_{d}\left(X_{i}, X_{j}\right)=\frac{1}{2}\left(a_{i j}+a_{j i}\right) .
\end{aligned}
$$

Thus, if we assume that $A$ takes its values in the subspace $\mathfrak{s} \mathfrak{D}(d, n-d)^{\mathrm{C}}$, then $X_{1}, \ldots, X_{n}$ constitutes a basis for $\mathscr{F}_{x}$ and the subbundle $\mathscr{F}$ is isotropic. A vector $Y$ belongs to $\mathscr{F}_{x}$ if and only if $g_{d}\left(Y, X_{s}\right)=0$ for all $s$.

If $f$ is a complex valued function on an open subset of $\mathbf{R}_{d}^{2 n}$ then the differential of $f$ vanishes on $\mathscr{F}$ if and only if $X_{s}(f)=0$ for all $s$, that is

$$
\begin{aligned}
-\partial_{v_{l}} f-\sum_{r=1}^{d} a_{l r} \partial_{u_{r}} f+\sum_{k=d+1}^{n} a_{l k} \partial_{q_{k}} f & =0 \\
\partial_{\bar{q}_{j}} f-\sum_{r=1}^{d} a_{j r} \partial_{u_{r}} f+\sum_{k=d+1}^{n} a_{j k} \partial_{q_{k}} f & =0,
\end{aligned}
$$

Proposition 3.2. Any locally defined complex valued function whose differential vanishes on $\mathscr{F}$ is horizontally conformal.

PROoF. If $f$ is a locally defined complex valued function with $d f=0$ on $\mathscr{F}$, then $d f$ is a linear combination of $\xi_{1}, \ldots, \xi_{n}$. Hence $\nabla f$ is a vector in $\mathscr{F}$ so $g_{d}(\nabla f, \nabla f)=0$ since $\mathscr{F}$ is isotropic. The result now follows from Proposition 2.4.

Finally, given the map $A$ with values in $\mathfrak{s} \mathfrak{D}(d, n-d)^{\mathrm{C}}$, we introduce the 
following functions:

$$
\begin{aligned}
& w_{l} \stackrel{\text { def }}{=} u_{l}+\sum_{r=1}^{d} a_{l r} v_{r}+\sum_{k=d+1}^{n} a_{l k} \bar{q}_{k}, \\
& w_{j} \stackrel{\text { def }}{=} q_{j}+\sum_{r=1}^{d} a_{j r} v_{r}+\sum_{k=d+1}^{n} a_{j k} \bar{q}_{k} .
\end{aligned}
$$

\section{Construction of harmonic morphisms}

From the results of the previous section, we now have means to construct isotropic subbundles of $T \mathrm{R}_{d}^{2 n}$. The following shows how to construct implicitly defined horizontally conformal maps using Proposition 3.2.

Theorem 4.1. Let $\Omega \subseteq \mathrm{C}^{n} \times \mathrm{C}^{m}$ and $U \subseteq \mathrm{C}^{m}$ be open sets and let

$$
f: \Omega \rightarrow \mathrm{C}^{m}, \quad A: U \rightarrow \mathfrak{s} \mathfrak{D}(d, n-d)^{\mathrm{C}}
$$

be holomorphic maps. Assume that

$$
F: W \subseteq \mathrm{R}_{d}^{2 n} \times \mathrm{C}^{m} \rightarrow \mathrm{C}^{m}, \quad F(x, z) \stackrel{\text { def }}{=} f(w(x, z), z),
$$

may be defined on a nonempty open set $W$ and choose $\left(x_{0}, z_{0}\right) \in W$ such that

$$
F\left(x_{0}, z_{0}\right)=0, \quad \operatorname{det}\left(\partial_{z^{\mu}} F^{\nu}\left(x_{0}, z_{0}\right)\right) \neq 0 .
$$

Then the components of the local solution $z=z(x)$ to the equation

$$
F(x, z)=0, \quad z\left(x_{0}\right)=z_{0},
$$

have their gradients in the subbundle determined by $A \circ z$. In particular, they are horizontally conformal. gives

Proof. Taking various derivatives of the equation $F(x, z)=f(w(x, z), z)$

$$
\begin{array}{ll}
\partial_{u_{l}} F^{v}=\partial_{w_{l}} f^{v}, & \partial_{v_{l}} F^{v}=\sum_{s=1}^{n} a_{s l} \partial_{w_{s}} f^{v}, \\
\partial_{q_{i}} F^{v}=\partial_{w_{i}} f^{v}, & \partial_{\bar{q}_{i}} F^{v}=\sum_{s=1}^{n} a_{s i} \partial_{w_{s}} f^{v} .
\end{array}
$$


Hence

$$
\begin{aligned}
& -\partial_{v_{l}} F^{v}-\sum_{r=1}^{d} a_{l r} \partial_{u_{r}} F^{v}+\sum_{k=d+1}^{n} a_{l k} \partial_{q_{k}} F^{v}=-\sum_{s=1}^{n} a_{s l} \partial_{w_{s}} f^{v}-\sum_{r=1}^{d} a_{l r} \partial_{w_{r}} f^{v} \\
& +\sum_{k=d+1}^{n} a_{l k} \partial_{w_{k}} f^{v}=0 \\
& \partial_{\bar{q}_{i}} F^{v}-\sum_{r=1}^{d} a_{i r} \partial_{u_{r}} F^{v}+\sum_{k=d+1}^{n} a_{i k} \partial_{q_{k}} F^{v}=\sum_{s=1}^{n} a_{s i} \partial_{w_{s}} f^{v}-\sum_{r=1}^{d} a_{i r} \partial_{w_{r}} f^{v} \\
& +\sum_{k=d+1}^{n} a_{i k} \partial_{w_{k}} f^{\mathcal{v}}=0
\end{aligned}
$$

Differentiating $F(x, z(x))=0$ with respect to $\rho=u_{1}, v_{1}, \ldots, q_{1}, \ldots, \bar{q}_{n}$ gives

$$
\partial_{\rho} F^{v}+\sum_{\mu=1}^{m} \partial_{z^{\mu}} F^{v} \partial_{\rho} z^{\mu}=0
$$

Solving this for $\partial_{\rho} z^{\mu}$ shows together with the above calculations that $z^{\mu}$ belongs to the subbundle for $\mu=1, \ldots, m$.

We thus have means to construct horizontally conformal maps. The next thing to do is to investigate the tension field of local solutions. An easy calculation shows that in the coordinates $\left(u_{1}, v_{1}, \ldots, q_{1}, \ldots, \bar{q}_{n}\right)$ the tension field of a function $f$ is given by

$$
\frac{1}{4} \tau(f)=-\sum_{r=1}^{d} \partial_{u_{r} v_{r}}^{2} f+\sum_{k=d+1}^{n} \partial_{q_{k} \bar{q}_{k}}^{2} f .
$$

Assume that $z=\left(z^{1}, \ldots, z^{m}\right)$ is a local solution as before. Since the gradient of $z^{1}$ belongs to the subbundle we know that

$$
\begin{aligned}
& \partial_{v_{l}} z^{1}=-\sum_{r=1}^{d} a_{l r} \partial_{u_{r}} z^{1}+\sum_{k=d+1}^{n} a_{l k} \partial_{q_{k}} z^{1}, \\
& \partial_{\bar{q}_{j}} z^{1}=\sum_{r=1}^{d} a_{j r} \partial_{u_{r}} z^{1}-\sum_{k=d+1}^{n} a_{j k} \partial_{q_{k}} z^{1} .
\end{aligned}
$$


Then we get

$$
\begin{aligned}
\frac{1}{4} \tau\left(z^{1}\right)= & -\sum_{r=1}^{d-1} \partial_{u_{r}}\left(-\sum_{l=1}^{d} a_{r l} \partial_{u_{l}} z^{1}+\sum_{k=d+1}^{n} a_{r k} \partial_{q_{k}} z^{1}\right) \\
& +\sum_{k=d+1}^{n} \partial_{q_{k}}\left(\sum_{r=1}^{d} a_{k r} \partial_{u_{r}} z^{1}-\sum_{i=d+1}^{n} a_{k i} \partial_{q_{i}} z^{1}\right) \\
= & \sum_{r, l=1}^{d} \partial_{u_{r}} a_{r l} \partial_{u_{l}} z^{1}+\sum_{r, l=1}^{d} a_{r b} \partial_{u_{r} u_{l}}^{2} z^{1} \\
& -\sum_{r=1}^{d} \sum_{k=d+1}^{n} \partial_{u_{r}} a_{r k} \partial_{q_{k}} z^{1}-\sum_{r=1}^{d} \sum_{k=d}^{n} a_{r k} \partial_{u_{r} q_{k}}^{2} z^{1} \\
& +\sum_{k=d+1}^{n} \sum_{r=1}^{d} \partial_{q_{k}} a_{k r} \partial_{u_{r}} z^{1}+\sum_{k=d+1}^{n} \sum_{r=1}^{d} a_{k r} \partial_{q_{k} u_{r}}^{2} z^{1} \\
& -\sum_{i, k=d+1}^{n} \partial_{q_{k}} a_{k i} \partial_{q_{i}} z^{1}-\sum_{i, k=d+1}^{n} a_{k i} \partial_{q_{k} q_{i}}^{2} z^{1} .
\end{aligned}
$$

Here, the second, fourth, sixth and eight term vanish since $A$ takes its values in $\mathfrak{S} \mathfrak{D}(d, n-d)^{\mathrm{C}}$. Assuming that $A=A(z)$ is holomorphic, this may be written as

$$
\begin{aligned}
\frac{1}{4} \tau\left(z^{1}\right)=\sum_{\nu=1}^{m} & \sum_{r, l=1}^{d} \partial_{z^{v}} a_{r l} \partial_{u_{r}} z^{\nu} \partial_{u_{l}} z^{1}-\sum_{\nu=1}^{m} \sum_{r=1}^{d} \sum_{k=d+1}^{n} \partial_{z^{v}} a_{r k} \partial_{u_{r}} z^{\nu} \partial_{q_{k}} z^{1} \\
& +\sum_{\nu=1}^{m} \sum_{k=d}^{n} \sum_{r=1}^{d} \partial_{z^{v}} a_{k r} \partial_{q_{k}} z^{\nu} \partial_{u_{r}} z^{1}-\sum_{\nu=1}^{m} \sum_{i, k=d+1}^{n} \partial_{z^{v}} a_{k i} \partial_{q_{k}} z^{\nu} \partial_{q_{i}} z^{1}
\end{aligned}
$$

Thus if we assume that $A$ only depends on $z^{1}$, then the first and last terms vanish due to skew-symmetry of the indices and the two middle terms cancel out each other. We have proven the following theorem.

THEOREM 4.2. Let $\Omega \subseteq \mathrm{C}^{n} \times \mathrm{C}^{m}$ and $U \subseteq \mathrm{C}^{m}$ be open sets and let

$$
f: \Omega \rightarrow \mathrm{C}^{m}, \quad A: U \rightarrow \mathfrak{G} \mathfrak{D}(d, n-d)^{\mathrm{C}}
$$

be holomorphic maps. Assume that

$$
F: W \subseteq \mathrm{R}_{d}^{2 n} \times \mathrm{C}^{m} \rightarrow \mathrm{C}^{m}, \quad F(x, z) \stackrel{\text { def }}{=} f(w(x, z), z),
$$


may be defined on a nonempty open set $W$. Suppose that $\left(x_{0}, z_{0}\right) \in W$ and $F\left(x_{0}, z_{0}\right)=0$. If $A$ only depends on $z^{1}$ and

$$
\operatorname{det}\left(\partial_{z^{\mu}} F^{v}\left(x_{0}, z_{0}\right)\right) \neq 0,
$$

then the first component of the local solution $z=z(x)$ to the equation

$$
F(x, z)=0, \quad z\left(x_{0}\right)=z_{0},
$$

is a harmonic morphism from a neighbourhood of $x_{0}$ in $\mathbf{R}_{d}^{2 n}$ into $C$. This holds in particular if $m=1$.

Remark 4.3. The above result is in a sense dual to Proposition 3.18 of [4]. If we interpret the complex subbundles constructed in this paper as a semiRiemannian version of almost Hermitian structures, then the fact that the map $A$ only depends on $z^{1}$ means that the structure is parallel along the fibres of $z^{1}$. This should be compared with the concept of superminimality [4, Section 4].

Example 4.4. Let $A: \mathrm{C} \rightarrow \mathfrak{s} \mathfrak{D}(2,2)^{\mathrm{C}}$ be defined by

$$
A(z)=\left(\begin{array}{rrrr}
0 & z & 0 & 0 \\
-z & 0 & 0 & 1 \\
0 & 0 & 0 & -1 \\
0 & 1 & 1 & 0
\end{array}\right),
$$

and take

$$
f: \mathrm{C}^{4} \times \mathbf{C} \rightarrow \mathrm{C}, \quad f\left(w_{1}, w_{2}, w_{3}, w_{4}, z\right)=z w_{1}^{3}+w_{2}^{2} w_{3}+w_{3}^{2} w_{4} .
$$

From equation (5), using the matrix $A$, we consider $\left(w_{1}, w_{2}, w_{3}, w_{4}\right)$ as functions of $\left(x_{1}, \ldots, x_{8}\right)$ and $z$. Thus, the local solution $z=z(x)$ to the equation

$$
z w_{1}^{3}+w_{2}^{2} w_{3}+w_{3}^{2} w_{4}=0, \quad z(-1,0, \ldots, 0)=0,
$$

is a harmonic morphism from an open neighbourhood of $(-1,0, \ldots, 0)$ in $\mathbf{R}_{2}^{8}$ to $\mathrm{C}$.

\section{Invariance of solutions}

Here we describe a method for constructing locally defined harmonic morphisms in $\mathrm{R}_{d}^{2 n-1}$. For this, assume that a holomorphic map

$$
A: U \subseteq \mathrm{C} \rightarrow \mathfrak{s} \mathfrak{D}(d, n-d)^{\mathrm{C}}
$$


is given. Define $w_{1}, \ldots, w_{n}$ as before and make the following change of variables:

$\tilde{w}_{1}=w_{1}+a_{1 n} w_{n}, \tilde{w}_{2}=w_{2}+a_{2 n} w_{n}, \ldots, \tilde{w}_{n-1}=w_{n-1}+a_{n-1 n} w_{n}, \tilde{w}_{n}=w_{n}$.

Note that $\tilde{w}_{1}, \ldots, \tilde{w}_{n-1}$ do not depend on $x_{2 n}$. Thus we have the following obvious result.

THeOREM 5.1. Let $\Omega \subseteq \mathrm{C}^{n-1} \times \mathrm{C}^{m}$ and $U \subseteq \mathrm{C}$ be open sets and let

$$
f: \Omega \rightarrow \mathrm{C}^{m}, \quad A: U \rightarrow \mathfrak{S} \mathfrak{D}(d, n-d)^{\mathrm{C}}
$$

be holomorphic maps. Assume that

$$
F: W \subseteq \mathrm{R}_{d}^{2 n-1} \times \mathrm{C}^{m} \rightarrow \mathrm{C}^{m}, \quad F(x, z) \stackrel{\text { def }}{=} f\left(\tilde{w}_{1}, \ldots, \tilde{w}_{n-1}, z\right),
$$

may be defined on a non-empty open set $W$ and choose $\left(x_{0}, z_{0}\right) \in W$ such that

$$
F\left(x_{0}, z_{0}\right)=0, \quad \operatorname{det}\left(\partial_{z^{\mu}} F^{\nu}\left(x_{0}, z_{0}\right)\right) \neq 0 .
$$

Then the first component $z^{1}$ of the local solution $z=z(x)$ to the equation

$$
F(x, z)=0, \quad z\left(x_{0}\right)=z_{0},
$$

is a harmonic morphism in an open subset of $\mathrm{R}_{d}^{2 n-1}$

To investigate whether or not a map $\phi$ is invariant along a vector field $\sum_{s} \zeta_{s} \partial_{x_{s}}$ in $\mathrm{R}_{d}^{2 n}$ one should study the equation

$$
\begin{aligned}
0=d \phi\left(\sum_{s} \zeta_{s} \partial_{x_{s}}\right)=\sum_{r=1}^{d} & \left(\zeta_{2 r-1}-\zeta_{2 r}\right) \partial_{u_{r}} \phi+\left(\zeta_{2 r-1}+\zeta_{2 r}\right) \partial_{v_{r}} \phi \\
& +\sum_{k=d+1}^{n}\left(\left(\zeta_{2 k-1}+i \zeta_{2 k}\right) \partial_{q_{k}} \phi+\left(\zeta_{2 k-1}-i \zeta_{2 k}\right) \partial_{\bar{q}_{k}} \phi\right) .
\end{aligned}
$$

Now suppose that $\phi=z^{1}$ is the first coordinate of a local solution to equation (6). Let $K=\left(\partial_{z^{\mu}} F^{v}\right)_{\nu, \mu}$. Then

$$
\partial_{\rho} z^{1}=-\sum_{\nu=1}^{m}\left(K^{-1}\right)_{1 \nu} \partial_{\rho} F^{v},
$$


for any $\rho=u_{1}, v_{1}, \ldots, q_{1}, \ldots, \bar{q}_{n}$. Equation (9) may then be written as

$$
\begin{aligned}
0=- & \sum_{\nu=1}^{m}\left(K^{-1}\right)_{1 v}\left(\sum_{r=1}^{d}\left(\zeta_{2 r-1}-\zeta_{2 r}\right) \partial_{u_{r}} F^{v}+\left(\zeta_{2 r-1}+\zeta_{2 r}\right) \partial_{v_{r}} F^{v}\right. \\
& \left.+\sum_{k=d+1}^{n}\left(\left(\zeta_{2 k-1}+i \zeta_{2 k}\right) \partial_{q_{k}} F^{v}+\left(\zeta_{2 k-1}-i \zeta_{2 k}\right) \partial_{\bar{q}_{k}} F^{v}\right)\right) .
\end{aligned}
$$

In particular, we wish to find conditions for invariance of solutions under the action of $\mathrm{R}^{*}$ on $\mathrm{R}_{d}^{2 n}$. The vector field in question is then $\sum_{s} x_{s} \partial_{x_{s}}$, so equation (11) reduces to

$0=-\sum_{v=1}^{m}\left(K^{-1}\right)_{1 v}\left(\sum_{r=1}^{d}\left(u_{r} \partial_{u_{r}} F^{v}+v_{r} \partial_{v_{r}} F^{v}\right)+\sum_{k=d+1}^{n}\left(q_{k} \partial_{q_{k}} F^{v}+\bar{q}_{k} \partial_{\bar{q}_{k}} F^{v}\right)\right)$.

Suppose that $f(w, z)$ is homogeneous in its first argument. Then equation (12) may be written as

$$
0=\sum_{\nu=1}^{m}\left(K^{-1}\right)_{1 v} F^{v}(x, z(x)),
$$

which is trivially satisfied since $F(x, z(x))=0$.

EXAMPLE 5.2. The harmonic morphism constructed in Example 4.4 factors by homogenity to a harmonic morphism from an open subset of $\mathrm{RH}_{2}^{7}$ to $\mathrm{C}$.

Thus, assuming homogenity in addition to the assumptions of Theorem 5.1 we get the following result.

Corollary 5.3. Let $\Omega \subseteq \mathrm{C}^{n-1} \times \mathrm{C}^{m}$ and $U \subseteq \mathrm{C}$ be open sets and let

$$
f: \Omega \rightarrow \mathrm{C}^{m}, \quad A: U \rightarrow \mathfrak{s} \mathfrak{D}(d, n-d)^{\mathrm{C}}
$$

be holomorphic maps, where $f$ is homogeneous in its first argument. Assume that

$$
F: W \subseteq \mathrm{R}_{d}^{2 n-1} \times \mathrm{C}^{m} \rightarrow \mathrm{C}^{m}, \quad F(x, z) \stackrel{\text { def }}{=} f\left(\tilde{w}_{1}, \ldots, \tilde{w}_{n-1}, z\right),
$$

may be defined on a non-empty open set $W$ and choose $\left(x_{0}, z_{0}\right) \in W$ such that

$$
F\left(x_{0}, z_{0}\right)=0, \quad \operatorname{det}\left(\partial_{z^{\mu}} F^{v}\left(x_{0}, z_{0}\right)\right) \neq 0 .
$$

Then the first component $z^{1}$ of the local solution $z=z(x)$ to the equation

$$
F(x, z)=0, \quad z\left(x_{0}\right)=z_{0},
$$


is a harmonic morphism in an open subset of $\mathrm{R}_{d}^{2 n-1}$ which is invariant under the action of $\mathbf{R}^{*}$ on $\mathbf{R}_{d}^{2 n-1}$. In particular, if $g_{d}\left(x_{0}, x_{0}\right)<0$, then $z$ induces a harmonic morphism in an open subset of $\mathrm{R} H_{d}^{2 n-2}$.

EXAMPLE 5.4. For $z \in \mathrm{C}$, let

$$
A(z)=\left(\begin{array}{rrrrrr}
0 & 1 & 0 & 0 & 0 & 0 \\
1 & 0 & 1 & 0 & 0 & 0 \\
0 & -1 & 0 & z & 0 & 1 \\
0 & 0 & -z & 0 & 0 & 0 \\
0 & 0 & 0 & 0 & 0 & -1 \\
0 & 0 & -1 & 0 & 1 & 0
\end{array}\right) \in \mathfrak{S}_{\mathcal{O}}(1,5)^{\mathrm{C}}
$$

Thus, according to (5) we define

$$
\begin{aligned}
w_{1}=u_{1}+\bar{q}_{2}, \quad w_{2}=q_{2}+v_{1}+\bar{q}_{3}, \quad w_{3}=q_{3}-\bar{q}_{2}+z \bar{q}_{4}+\bar{q}_{6}, \\
w_{4}=q_{4}-z \bar{q}_{3}, \quad w_{5}=q_{5}-\bar{q}_{6}, \quad w_{6}=q_{6}-\bar{q}_{3}+\bar{q}_{5} .
\end{aligned}
$$

Take

$$
f=f\left(\tilde{w}_{1}, \tilde{w}_{2}, \tilde{w}_{3}, \tilde{w}_{4}, \tilde{w}_{5}, z\right)=e^{z^{2} \tilde{w}_{2} / \tilde{w}_{1}} \tilde{w}_{5}^{2}+e^{z\left(\tilde{w}_{3} / \tilde{w}_{1}\right)^{2}} \tilde{w}_{4}^{2}+(z-1) \tilde{w}_{1}^{2},
$$

and change variables from $w_{1}, \ldots, w_{6}$ to $\tilde{w}_{1}, \ldots, \tilde{w}_{6}$ as describes above. If we define $F(x, z)=f(\tilde{w}(x, z), z)$, then the local solution to the equation

$$
F(x, z)=0, \quad z(-1,0, \ldots, 0)=1
$$

will define a local harmonic morphism in $\mathrm{R} H^{10}$.

EXAMPLE 5.5. Let

$$
A=\left(\begin{array}{rrrr}
0 & 0 & 0 & 0 \\
0 & 0 & -1 & 1 \\
0 & 1 & 0 & i \\
0 & -1 & -i & 0
\end{array}\right) \in \mathfrak{S} \mathfrak{D}(1,3)^{\mathrm{C}},
$$

Study a solution $z$ to the equation

$$
z^{2} \tilde{w}_{1}^{3}-2 z \tilde{w}_{3}^{2} \tilde{w}_{2}+\tilde{w}_{3} \tilde{w}_{2}^{2}=0
$$

i.e.

$$
z=\frac{w_{2}+w_{4}}{w_{1}^{3}}\left(\left(w_{3}+i w_{4}\right)^{2} \pm \sqrt{\left(w_{3}+i w_{4}\right)^{4}-\left(w_{3}+i w_{4}\right) w_{1}^{3}}\right) .
$$


Here, as before,

$$
\begin{aligned}
& w_{1}=x_{1}-x_{2} \\
& w_{2}=x_{3}+i x_{4}-x_{5}+i x_{6}+x_{7}-i x_{8} \\
& w_{3}=x_{5}+i x_{6}+x_{3}-i x_{4}+i x_{7}+x_{8} \\
& w_{4}=x_{7}+i x_{8}-x_{3}+i x_{4}-i x_{5}-x_{6} .
\end{aligned}
$$

Given suitable initial conditions, this will define a harmonic morphism in an open subset of $\mathrm{R} H^{6}$.

EXAMPLE 5.6. We show how to construct a globally defined harmonic morphism in $\mathrm{RH}^{6}$ by finding an $\mathrm{R}^{*}$-invariant harmonic morphism in $\mathrm{R}_{1}^{8}$ which does not depend on $x_{8}$. For this, take $A$ to be the matrix

$$
A=\left(\begin{array}{rrrr}
0 & 0 & 0 & 0 \\
0 & 0 & -1 & 1 \\
0 & 1 & 0 & 0 \\
0 & -1 & 0 & 0
\end{array}\right) \in \mathfrak{s} \mathfrak{0}(1,3)^{\mathrm{C}}
$$

By (5), this means that

$$
\begin{aligned}
& w_{1}=u_{1} \\
& w_{2}=q_{2}-\bar{q}_{3}+\bar{q}_{4} \\
& w_{3}=q_{3}+\bar{q}_{2} \\
& w_{4}=q_{4}-\bar{q}_{2} .
\end{aligned}
$$

If $f$ is given by

$$
f(w, z)=z w_{1}^{2}-w_{3}\left(w_{2}+w_{4}\right)=z \tilde{w}_{1}^{2}-\tilde{w}_{3} \tilde{w}_{2},
$$

and we define as before $F(x, z)=f(w(x), z)$, then the local solution to $F(x, z)=0$ is given by

$$
z=\frac{w_{3}\left(w_{2}+w_{4}\right)}{w_{1}^{2}}=\frac{\tilde{w}_{3} \tilde{w}_{2}}{\tilde{w}_{1}^{2}}=\frac{\left(\bar{q}_{2}+q_{3}\right)\left(q_{2}-\bar{q}_{2}-\bar{q}_{3}+q_{4}+\bar{q}_{4}\right)}{u_{1}^{2}} .
$$

By Corollary 5.3, $z$ defines a globally defined harmonic morphism in $\mathrm{R} H^{6}$

Remark 5.7. In the above example we have chosen the matrix $A$ to be constant. If the corresponding complex subbundle is interpreted as a semiRiemannian almost Hermitian structure as in Remark 4.3, then $A$ constant would mean that the structure in some sense is Kähler. It is not known if it is possible to construct a globally defined harmonic morphism as above 
without using a constant matrix. It would be interesting to know if there is some "Bernstein-type" result of this kind.

ACKNOWLeDgements. I am deeply indebted to S. Gudmundsson and J. C. Wood for their helpful comments and engagement in the questions and results of this paper.

\section{REFERENCES}

1. Baird, P., Riemannian twistors and Hermitian structures on low-dimensional space forms, J. Math. Phys. 33(10) (1992), 3340-3355.

2. Baird, P. and Wood, J. C., Harmonic morphisms between Riemannian manifolds, to appear.

3. Baird, P. and Wood, J. C., Harmonic morphisms and conformal foliations by geodesics of three-dimensional space forms, J. Austral. Math. Soc. Ser. A 51(1) (1991), 118-153.

4. Baird, P. and Wood, J. C., Hermitian structures and harmonic morphisms in higher-dimensional Euclidean spaces, Internat. J. Math. 6(2) (1995), 161-192.

5. Baird, P. and Wood, J. C., Weierstrass representations for harmonic morphisms on Euclidean spaces and spheres, Math. Scand. 81(2) (1997), 283-300.

6. Fuglede, B., Harmonic morphisms between Riemannian manifolds, Ann. Inst. Fourier (Grenoble) 28(2) (1978), vi, 107-144.

7. Fuglede, B., Harmonic morphisms between semi-Riemannian manifolds, Ann. Acad. Sci. Fenn. Math. 21(1) (1996), 31-50.

8. Gudmundsson, S., The Bibliography of Harmonic Morphisms, http : //www . maths. Ith. se/ matematiklu/personal/sigma/harmonic/bibliography.html.

9. Gudmundsson, S., Minimal submanifolds of hyperbolic spaces via harmonic morphisms, Geom. Dedicata 62(3) (1996), 269-279.

10. Gudmundsson, S., On the existence of harmonic morphisms from symmetric spaces of rank one, Manuscripta Math. 93(4) (1997), 421-433.

11. O’Neill, B., Semi-Riemannian Geometry, Academic Press Inc. [Harcourt Brace Jovanovich Publishers], New York, 1983, with applications to relativity.

12. Wolf, J. A., Spaces of constant curvature, Publish or Perish Inc., Houston, TX, fifth edition, 1984.

13. Wood, J. C., Harmonic morphisms and Hermitian structures on Einstein 4-manifolds, Internat. J. Math. 3(3) (1992), 415-439.

CENTRE FOR MATHEMATICAL SCIENCES

LUND UNIVERSITY - BOX 118

22100 LUND

SWEDEN

E-mail: Martin.Svensson@math.lu.se 